\title{
Gaze Estimation Model for Eye Drawing
}

\section{Alvin W. Yeo}

Faculty of Computer Science and

Information Technology

Universiti Malaysia Sarawak

94300 Kota Samarahan

Sarawak, Malaysia

alvin@fit.unimas.my

\section{Po-Chan Chiu}

Faculty of Computer Science and

Information Technology

Universiti Malaysia Sarawak

94300 Kota Samarahan

Sarawak, Malaysia

pcchiu@fit.unimas.my

\section{Abstract}

This paper describes a model that can be employed in eye drawing software applications. Unlike most of the existing interfaces for eye typing, eye drawing focuses on small target selection and moves the cursor to a precise location. This is made possible by a proposed Gaze Estimation Model which interprets users' interest when they want to draw new objects in a particular position.

\section{Keywords}

Eye tracking, eye movement, drawing, input devices, interaction techniques, assistive technology.

\section{ACM Classification Keywords}

H.5.2. User Interfaces: Input devices and strategies, interaction styles.

\section{Introduction}

Gaze-controlled interfaces constitute a new area of user interfaces intended for the community of able-bodied and disabled people. This area has attracted much interest from $\mathrm{HCI}$ researchers $[3,4,5,8,9]$

Many research works in gaze-controlled interfaces have focused on target selection with eye gaze. For example, Salvucci [6] developed a method for eye typing based on gaze patterns and language models. However, the method in target selection cannot be completely applied to other domains. Unlike target selection in eye typing, eye drawing's target selection is very small and the 